\title{
Anhang, mit Wolfram Mathematica erstellt
}

(*Definition der Dichtefunktion*)

$\mathrm{f}\left[\mathrm{x}_{-}, \mathrm{r}_{-}, \mathrm{m}_{-}, \mathrm{s}_{-}\right]:=1 / \mathrm{Sqrt}\left[2 * \mathrm{Pi}^{*} \mathrm{~s}^{\wedge} 2(1-\mathrm{r} *(\mathrm{x}-\mathrm{m}))\right]^{*} \operatorname{Exp}\left[-(\mathrm{x}-\mathrm{m})^{\wedge} 2 /\left(2 * \mathrm{~s}^{\wedge} 2\left(1-\mathrm{r}^{*}\right.\right.\right.$ $(\mathrm{x}-\mathrm{m})))$ ]

(* Es ist tatsächlich eine Dichte im Fall $r>0 *$ )

Integrate[f[x,r,m,s],\{x,-Infinity, $1 / \mathrm{r}+\mathrm{m}\}$, Assumptions- $>\{\mathrm{r}>0, \mathrm{~s}>0\}]$

1

(* Es ist tatsächlich eine Dichte auch im Fall $\mathrm{r}<0 *$ )

Integrate $[\mathrm{f}[\mathrm{x}, \mathrm{r}, \mathrm{m}, \mathrm{s}],\{\mathrm{x}, 1 / \mathrm{r}+\mathrm{m}$, Infinity $\}$, Assumptions $->\{\mathrm{r}<0, \mathrm{~s}>0\}]$

1

(* Erwartungswert $\mathrm{E}[\mathrm{X}]$ im Fall $\mathrm{r}>0, \mathrm{~m}=0$ mit $\mathrm{m}$ ungleich 0 kommt einfach + m dazu*)

Integrate[f[x,r,0,s]*x, $\{\mathrm{x},-$ Infinity, $1 / \mathrm{r}\}$, Assumptions- $>\{\mathrm{r}>0, \mathrm{~s}>0\}]$

$-\mathrm{rs} 2$

(* Erwartungswert $\mathrm{E}[\mathrm{X}]$ im Fall $\mathrm{r}<0, \mathrm{~m}=0$ ist aus Symmetriegründen auch $\left.-\mathrm{rs}^{\wedge} 2 *\right)$

Integrate $\left[\mathrm{f}[\mathrm{x}, \mathrm{r}, 0, \mathrm{~s}]^{*} \mathrm{x},\{\mathrm{x}, 1 / \mathrm{r}\right.$, Infinity $\}$, Assumptions- $\left.>\{\mathrm{r}<0, \mathrm{~s}>0\}\right]$

(* Erwartungswert $\mathrm{E}\left[\mathrm{X}^{\wedge} 2\right]$. Wird benötigt für die Varianz, denn $\left.\operatorname{Var}[\mathrm{X}]=\mathrm{E}\left[\mathrm{X}^{\wedge} 2\right]-(\mathrm{E}[\mathrm{X}])^{\wedge} 2 .^{*}\right)$

Integrate $[\mathrm{f}[\mathrm{x}, \mathrm{r}, 0, \mathrm{~s}]]^{*} \mathrm{x}^{\wedge} 2,\{\mathrm{x}, 1 / \mathrm{r}$, Infinity $\}$, Assumptions- $\left.>\{\mathrm{r}<0, \mathrm{~s}>0\}\right]$

(* Erwartungswert $\mathrm{E}\left[\mathrm{X}^{\wedge} 2\right]$. Wird benötigt für die Varianz, denn $\operatorname{Var}[\mathrm{X}]=\mathrm{E}\left[\mathrm{X}^{\wedge} 2\right]-(\mathrm{E}[\mathrm{X}])^{\wedge} 2$. Fall $\mathrm{r}>0, \mathrm{~s}>0$ konvergiert $\left.*\right)$

Integrate $\left[\mathrm{f}[\mathrm{x}, \mathrm{r}, 0, \mathrm{~s}]^{*} \mathrm{x}^{\wedge} 2,\{\mathrm{x},-\right.$ Infinity, $1 / \mathrm{r}\}$, Assumptions- $\left.>\{\mathrm{r}>0, \mathrm{~s}>0\}\right]$

$\mathrm{s} 2+3 \mathrm{r} 2 \mathrm{~s} 4$

(* Wieder aus Symmetriegründen gilt auch im ersten Fall und $\mathrm{m}=0$ $\mathrm{E}\left[\mathrm{X}^{\wedge} 2\right]=\mathrm{s}^{\wedge} 2+3 \mathrm{r}^{\wedge} 2 \mathrm{~s}^{\wedge} 4$.

Die Varianz ist dann (diese ist unabhängig von $\mathrm{m}$ ) gegeben durch $\operatorname{Var}[\mathrm{X}]=\mathrm{E}[$ $\left.\left.X^{\wedge} 2\right]-(E[X])^{\wedge} 2=s^{\wedge} 2+3 r^{\wedge} 2 s^{\wedge} 4-\left(-r^{\wedge} 2\right)^{\wedge} 2=s^{\wedge} 2+2 r^{\wedge} 2 s^{\wedge} 4 *\right)$ 